\title{
Handheld high-throughput plasmonic biosensor using computational on-chip imaging
}

\author{
Arif E Cetin $^{1 *}$, Ahmet F Coskun ${ }^{2,3}$, Betty C Galarreta ${ }^{1,4}$, Min Huang ${ }^{1}$, David Herman ${ }^{2}$, Aydogan Ozcan ${ }^{2,6}$ \\ and Hatice Altug ${ }^{1,5}$
}

We demonstrate a handheld on-chip biosensing technology that employs plasmonic microarrays coupled with a lens-free computational imaging system towards multiplexed and high-throughput screening of biomolecular interactions for point-of-care applications and resource-limited settings. This lightweight and field-portable biosensing device, weighing $60 \mathrm{~g}$ and $7.5 \mathrm{~cm}$ tall, utilizes a compact optoelectronic sensor array to record the diffraction patterns of plasmonic nanostructures under uniform illumination by a single-light emitting diode tuned to the plasmonic mode of the nanoapertures. Employing a sensitive plasmonic array design that is combined with lens-free computational imaging, we demonstrate label-free and quantitative detection of biomolecules with a protein layer thickness down to $3 \mathrm{~nm}$. Integrating large-scale plasmonic microarrays, our on-chip imaging platform enables simultaneous detection of protein mono- and bilayers on the same platform over a wide range of biomolecule concentrations. In this handheld device, we also employ an iterative phase retrieval-based image reconstruction method, which offers the ability to digitally image a highly multiplexed array of sensors on the same plasmonic chip, making this approach especially suitable for high-throughput diagnostic applications in field settings.

Light: Science \& Applications (2014) 3, e122; doi:10.1038/Isa.2014.3; published online 3 January 2014

Keywords: computational imaging; high-throughput biodetection; lens-free imaging; on-chip sensing; plasmonics; point of care diagnostics; telemedicine

\section{INTRODUCTION}

Early detection and effective diagnosis are important for disease screening and preventing epidemics. Most of the current medical technologies are time-consuming and require costly chemical procedures and bulky instrumentation, necessitating an advanced medical infrastructure and trained laboratory professionals. In developing countries, there is an urgent need for cost-effective and easy-to-use diagnostics technologies. Similarly, in developed countries, even though resources are available, the cost of health care is still a concern. For various diseases including Alzheimer's disease and cancer, expression of certain proteins in the body is up- or downregulated, making them suitable to be used as disease biomarkers. Recent works have also shown that instead of monitoring a single biomarker, screening a panel of proteins could significantly improve the accuracy in medical diagnostics by eliminating false positives. ${ }^{1-3}$ High-throughput sensing technologies that can enable simultaneous detection of a wide range of proteins might offer a viable solution for medical diagnosis. Therefore, there is a strong need for affordable and highthroughput screening technologies that can effectively monitor and diagnose various medical conditions. As recognition of some of these challenges, international consortiums are calling for innovative solutions toward cost-effective and handheld wireless biosensor devices with a desired list of performance metrics (see, for example, The Qualcomm Tricorder X Prize and Nokia Sensing X Challenge). Similarly, various point-of-care diagnostic devices have been developed $^{4-29}$ and among them optical imaging and sensing techniques are highly advantageous as they can provide real-time, highresolution and highly sensitive quantitative information, potentially assisting rapid and accurate diagnosis. ${ }^{30-40}$ To date, a number of optical techniques have been proposed for point-of-care diagnostics such as in vitro optical devices, ${ }^{41-53}$ including portable optical imaging systems, optical microscopes integrated to cell phones or in vivo optical devices, ${ }^{54-63}$ involving confocal microscopy, microendoscopy and optical coherence tomography techniques. Among these approaches, lens-free computational on-chip imaging ${ }^{64}$ has been an emerging technique that can eliminate the need for bulky and costly optical components while also preserving (or even enhancing in certain cases) the image resolution, field of view and sensitivity. In this

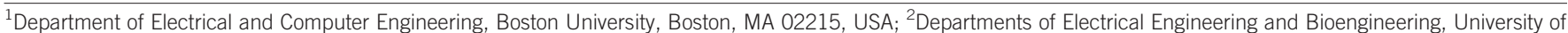

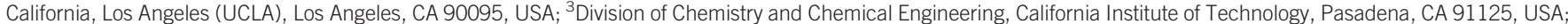

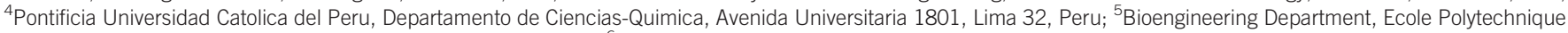

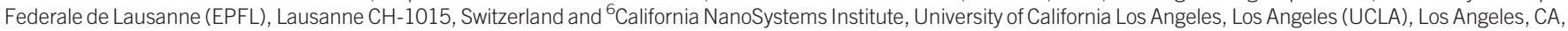
90095, USA

*These authors contribute equally to this work.

Correspondence: Professor A Ozcan, Departments of Electrical Engineering and Bioengineering, UCLA, Los Angeles, CA 90095, USA

E-mail: ozcan@ucla.edu

Or Professor H Altug, Bioengineering Department, Ecole Polytechnique Federale de Lausanne (EPFL), Lausanne CH-1015, Switzerland

E-mail: hatice.altug@epfl.ch

Received 8 April 2013; Revised 21 August 2013; Accepted 27 August 2013 
on-chip microscopy platform, computational holographic reconstruction and phase recovery methods are used to partially eliminate diffraction effects, providing higher resolution microscopic images across very large imaging areas, e.g., $>20-30 \mathrm{~mm}^{2}$ using off-the-shelf CMOS (Complementary Metal-Oxide-Semiconductor) imager chips. ${ }^{65,66}$ Together with the improvements provided by unique sample preparation and self-assembly techniques, lens-free on-chip imaging can even detect single viruses and sub-100 nm particles ${ }^{67,68}$ across a wide field of view, e.g., $>20 \mathrm{~mm}^{2}$ and provides a highthroughput nano-imaging platform.

In parallel to these advancements in computational imaging, current trends for health-care technologies aim to utilize high-throughput and massively multiplexed detection methods that can be used to rapidly extract a wide range of diagnostic data. ${ }^{69-73}$ One way of such multiplexing is fluorescence imaging. ${ }^{74-76}$ However, this approach suffers from cross-interference of labels with other molecular binding interactions, a limited number of fluorophore labels and additional labeling steps that increase the sample preparation time. Recently, various high-throughput optical detection methods have been investigated to overcome these problems as they offer strong advantages by being compatible with physiological solutions, not affected by the variation in the ionic strengths of biosolutions, and reducing sample contamination via allowing remote transduction of the biomolecular binding signal. ${ }^{77-85}$ Among optical biosensors, surface plasmon resonance (SPR)-based platforms are one of the most favored. Surface plasmons (SPs) are waves propagating at a metal/dielectric interface associated with the collective electron oscillations. This feature makes SPs highly suitable for investigation of near-field variations of a specimen due to absorption of specific molecules onto the metal surface, enabling labelfree detection of binding events. ${ }^{86-88}$ In fact, SPR is considered as the gold standard for label free biodetection. Strong sensitivities of SPR sensors allow detection of small proteins with molecular weights down to tens of $\mathrm{kDa} .{ }^{89,90}$ As gold is highly biocompatible, SPR sensors also enable robust surface chemistry and biofunctionalization necessary for specific detection of target biomolecules. However, multiplexing capability of conventional SPR sensors is limited in field settings due to the alignment sensitive prism coupling scheme and bulky instrumentation. These requirements pose major obstacles in achieving handheld diagnostics and health-care technologies. Recently, nanoplasmonics has taken significant attention as it can potentially overcome these challenges. ${ }^{9-94}$ Nanoscale plasmonic structures consisting of metallic particles and/or apertures provide new avenues for biosensing and spectroscopy due to their ability to generate dramatic field enhancements and spatially confine light on the nanometer scale. ${ }^{95-103}$ In particular, nanoaperture arrays support extraordinary optical transmission through the exploitation of plasmonic modes excited by the grating orders of the array. ${ }^{104,105}$ These plasmonic modes are highly sensitive to minute changes in the near-field refractive index of the nanoaperture. ${ }^{91-94}$ In addition, this grating configuration allows excitation light to be coupled to the SP waves even at normal incidence. This collinear configuration is naturally compatible for imaging within an array format, making it a competitive candidate for high-throughput sensing and diagnostics applications. ${ }^{106}$

In this letter, we introduce an integrated system that combines wide-field plasmonic arrays with lens-free computational on-chip imaging enabling a high-throughput and handheld label-free sensing device. Our lens-free computational platform utilizes a CMOS imager chip to record the diffraction patterns of the plasmonic structures without the use of any lenses under uniform illumination by a single light-emitting diode (LED) tuned to the plasmonic modes of the nano-apertures. In this system, the plasmonic sensor chip, the LEDbased excitation source and the compact CMOS image sensor are automatically aligned in a dark environment, without the need for any bulky optical instruments or mechanical microstages. Unlike existing multiplexed biodetection systems, this on-chip biosensing technology is ultracompact and light-weight $(\sim 7.5 \mathrm{~cm}$ tall and 60 g), making it highly suitable for field medicine and diagnostic needs. Our biosensor detects protein monolayers down to $3 \mathrm{~nm}$ thickness without any labels and enables quantitative analysis of protein binding events over a wide range of biomolecule concentrations. The multiplexing capability of our lens-free computational sensing system is demonstrated by simultaneous identification of protein mono- and bilayers on the same plasmonic chip. Employing a computational image reconstruction method that is based on iterative phase retrieval, our device provides a promising platform for high-throughput biosensing of for example over 150000 sensors on large-scale plasmonics chip through a CMOS imager with an active area of $5.7 \mathrm{~mm} \times 4.3 \mathrm{~mm}$.

As shown in Figure 1a and 1b, our on-chip plasmonic biosensing platform is comprised of the following components: (i) a plasmonic chip containing microarray pixels composed of periodic nanoholes fabricated on a thin gold film to detect the variations in the surface conditions (near-field) due to the specific binding of molecules on the chip surface. Scanning electron microscope (SEM) image of 6 plasmonic sensor pixels containing nanohole arrays is provided in Figure 1c; (ii) an LED source that significantly overlaps with the plasmonic modes of the nanostructured surface of the chip. These plasmonic nanohole arrays, when illuminated by an LED (peak wavelength: $\sim 683 \mathrm{~nm}$, bandwidth: $\sim 26 \mathrm{~nm}$ ), excite a plasmonic mode which supports electromagnetic fields strongly localized around the rims of the nanoholes at the top surface. The fields extend into the sensing medium, together with $\sim 550$ times intensity enhancement of as verified by our finite-difference time-domain simulation (FDTD) results shown in Figure 1d (see Supplementary Information for details); and (iii) a CMOS imager chip that records the diffraction patterns of the plasmonic nanoapertures, modulated by molecular binding events on the aperture surface. Design and fabrication details of this platform are presented in the next section, which is then followed by the section on 'results and discussion'.

\section{MATERIALS AND METHODS}

The presented computational biosensing platform combines widefield nanoplasmonic arrays with a lens-free on-chip imaging, where a CMOS imager chip records the diffraction patterns of the plasmonic structures without the use of any lenses under uniform illumination provided by a LED that overlaps with the plasmonic modes of the nanoapertures. Integrating the plasmonic sensor chip, the LED-based excitation source and the compact image sensor in a lightweight handheld unit, this biosensor platform can monitor multiple protein binding events without any labels at the point of care or in field settings.

\section{Design of the wide-field plasmonic microarrays}

In our platform, we utilize microarray pixels composed of nanoapertures in the form of nanohole arrays exhibiting multiple spectral points of extraordinary light transmission due to the excitation of SP waves at different grating orders (see Supplementary Information for the theory of the SP excitation through periodic structures and Supplementary Fig. S1 for the fabrication of nanohole arrays). ${ }^{106-111}$ For our analysis, we focus on the $\mathrm{SP}(-1,0)$ mode (indicated by a black arrow in experimental spectrum taken by an optical spectrum analyzer shown in Figure 2a), since it supports large transmission intensity which can be easily detected by our lens-free imaging device. Inset in Figure 2a shows 

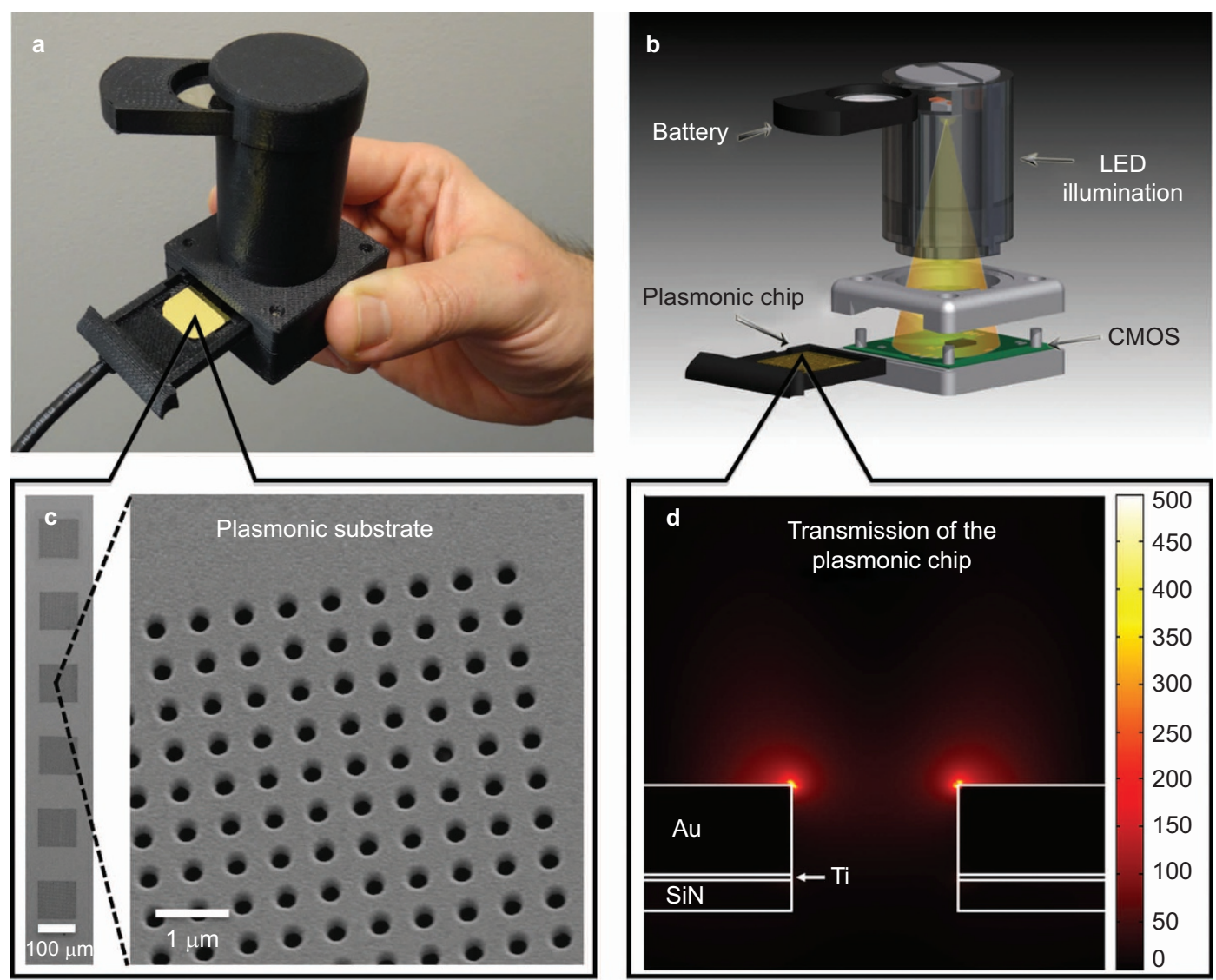

Figure 1 On-chip sensing platform with plasmonic microarrays and lens-free computational imaging. (a) Real picture of the portable biosensing device, weighing $60 \mathrm{~g}$ and $7.5 \mathrm{~cm}$ tall designed for point-of-care applications. In the picture, the hand of the author highlights the compactness of the device. (b) Schematic of the on-chip computational biosensing platform comprising a battery, an LED, a plasmonic chip and a CMOS imager chip. (c) SEM image of 6 plasmonic sensor pixels of size $100 \mu \mathrm{m} \times 100 \mu \mathrm{m}$ (the distance between plasmonic pixels are $100 \mu \mathrm{m}$ ) and the zoomed image of a plasmonic pixel containing periodic nanohole array. The diameter of the aperture is $200 \mathrm{~nm}$ and the period of the array is $600 \mathrm{~nm}$. (d) FDTD simulation of the near-field intensity enhancement distribution $\left(|E|^{2} /\left|E_{0}\right|^{2}\right)$ for the nanohole array calculated at the peak wavelength of the LED spectrum. The nanohole apertures are fabricated through $120 \mathrm{~nm}$ thick gold, $5 \mathrm{~nm}$ thick titanium and $80 \mathrm{~nm}$ thick silicon nitride layers. FDTD, finite-difference time-domain; LED, light emitting diode; SEM, scanning electron microscope.

the calculated distribution of the $y$-component of the magnetic field intensity, $\left(\left|H_{y}\right|^{2}\right)$. Here, the standing field pattern along the $x$-direction is due to the propagating SP excitation and the hot spots around the rims of the nanoholes along the $y$-direction are due to the localized SP waves. ${ }^{112,113}$ For the $\mathrm{SP}(-1,0)$ mode, the local excitations have dipolar character allowing the light to strongly couple to localized SPs resulting in an extraordinary light transmission. Localized SPs also lead to large near-field enhancements around the nanoapertures. Accordingly, our plasmonic aperture system shows high sensitivities to surface conditions by enabling strong overlap between analytes in the vicinity of the sensor surface (near-field) and the local fields. Upon biofunctionalization of the microarray pixels with a protein bilayer, containing $0.5 \mathrm{mg} \mathrm{mL} \mathrm{m}^{-1}$ protein $\mathrm{A} / \mathrm{G}$ and $0.5 \mathrm{mg} \mathrm{mL}^{-1}$ protein $\mathrm{IgG}$, which increases the local refractive index around the sensor surface, the plasmonic mode shifts to longer wavelengths by $\sim 19 \mathrm{~nm}$ (from $683 \mathrm{~nm}$ to $702 \mathrm{~nm}$ ). ${ }^{91,92}$ Protein $\mathrm{A} / \mathrm{G}$ is a recombinant fusion protein constituting binding domains of proteins $A$ and $G$. Protein A/G has a molecular weight of $50.46 \mathrm{kDa}$ and the thickness of protein $\mathrm{A} / \mathrm{G}$ layer is approximately $3 \mathrm{~nm} .{ }^{93}$ Protein IgG is immobilized on protein A/G due to the high affinity of protein $\mathrm{A} / \mathrm{G}$ to the $\mathrm{F} c$ regions of protein IgG. Molecular weight of protein IgG is $160 \mathrm{kDa}$ and the thicknesses of this layer is approximately $5 \mathrm{~nm}$ (see Supplementary Information for methods on protein chemistry and sample preparation techniques).

\section{Characterization of the lens-free on-chip sensing platform}

In our lens-free plasmonic sensing platform, the spectral shift in response to molecular binding is determined by monitoring the intensity changes captured by the CMOS imager (Figure 1). In order to reliably detect the red shift in the transmission resonance, the selection of the LED peak wavelength is critical, and it should ideally match to $683 \mathrm{~nm}$. As shown in Figure 2c, our LED response spectrally overlaps with the transmission resonance of the nanohole array located at $683 \mathrm{~nm}$. Figure $2 \mathrm{~b}$ illustrates that the presence of the protein binding events leads to a redshift in the transmission resonance, detuning it from the LED peak wavelength. Consequently, the total transmitted signal of the acquired lens-free image drops considerably; thus enabling to detect presence of biomolecular layers with a thickness down to $3 \mathrm{~nm}$ (refer to the section on 'results and discussion' for further details).

In our on-chip detection platform, the vertical distance between the plasmonic microarray pixel (with an individual pixel size of $100 \mu \mathrm{m} \times 100 \mu \mathrm{m}$ ) and the CMOS active area is $\sim 1 \mathrm{~mm}$. Therefore, even if the plasmonic interaction occurs in the near-field of the sensor chip, evanescent wave contribution is negligible during the detection or sampling of the lens-free diffraction patterns of the nanoapertures. For an accurate calculation for the diffraction pattern, we utilize an approach based on convolution and Fresnel kernel (see Figure 2d and Supplementary Fig. S2 for details of the field transmission calculations 


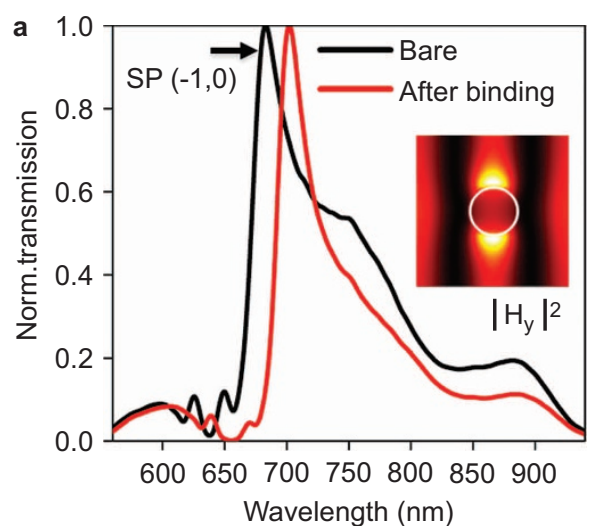

c

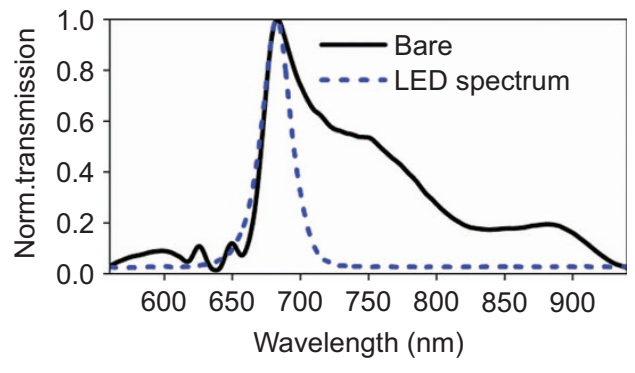

b
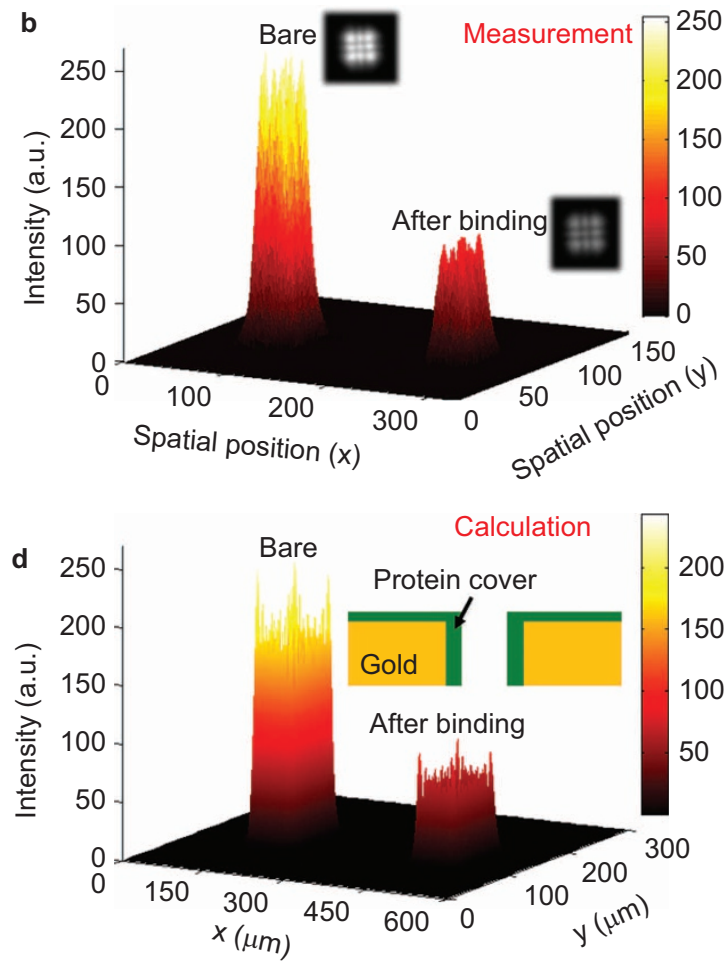

Figure 2 Working principle of our on-chip computational biosensing platform. (a) Experimental transmission spectra of the bare nanohole array (black solid line) and the plasmonic substrate covered with protein bilayer containing $0.5 \mathrm{mg} \mathrm{mL} \mathrm{L}^{-1} \mathrm{~A} / \mathrm{G}$ and $0.5 \mathrm{mg} \mathrm{mL} \mathrm{m}^{-1} \mathrm{gG}$ (red solid line). (Inset) FDTD simulation result showing the intensity distribution of the $y$-component of the magnetic field $\left(\left|H_{y}\right|^{2}\right)$ calculated for a bare aperture array at 683 nm. (b) 3D intensity plot of the experimental transmission signal and (inset) corresponding diffraction patterns of a plasmonic microarray pixel before and after the addition of the protein bilayer acquired by the CMOS imager. (c) Spectral curve demonstrating the overlap of the LED spectrum (blue dashed line) and the transmission response of the bare nanohole array (black solid line) measured by an optical spectrum analyzer. (d) 3D intensity plot of the simulated transmission pattern for the bare and the biofunctionalized nanohole array calculated at a propagation distance of $1 \mathrm{~mm}$ from the nanohole array structure to the sensor plane at the peak wavelength of the LED spectrum. CMOS, Complementary Metal-Oxide-Semiconductor; 3D, three-dimensional; FDTD, finite-difference time-domain; LED, light-emitting diode.

through our plasmonic chip). ${ }^{114}$ It is also important to note that since we utilize circular-shape apertures which have azimuthal symmetry in the aperture plane, the transmission response is the same for different linear polarization components (see Supplementary Fig. S3 for the near-field analysis of polarized vs. unpolarized light sources for our nanoaperture design). This behavior is highly advantageous for our lens-free computational detection system since a simple LED without a polarizer can be used for partially coherent illumination of the nanoaperture plane.

Computational reconstruction method for multiplexed plasmonic microarrays

To get multiplexed and high-throughput read-out, using a large number of sensor pixels into the same chip area results in a significant spatial overlap of diffraction patterns at the detector plane due to the lens-free operation. As illustrated in Figure 3a, the transmitted fields propagate $\sim 1 \mathrm{~mm}$ before being captured by the CMOS imager chip. To address this spatial overlap problem for especially high-density microarrays, we employ a numerical approach that is based on a phase recovery technique ${ }^{65}$ to digitally backpropagate the diffraction patterns onto the exit aperture of the nanohole array. ${ }^{114}$ We should emphasize that even using an LED, the spatial coherence diameter at the sensor chip surface can be fine-tuned between $0.2 \mathrm{~mm}$ and $1 \mathrm{~mm}$ by for example adjusting the vertical distance between the LED and sensor chip or changing the pinhole diameter in front of the LED. This partial spatial coherence ensures that each element of the plasmonic microarray effectively faces a quasimonochromatic and spatially coherent plane wave, such that we can frame the entire reconstruction process around phase recovery. The workflow of our iterative phase retrieval method (Figure 3b) can be summarized as follows: (i) lens-free diffraction images of the plasmonic microarrays are recorded using a CMOS imager chip; (ii) the raw diffraction images are then upsampled typically by a factor of $4-6$, using cubic spline interpolation method before the iterative reconstruction procedure. Note that this upsampling step does not increase the information content of the diffraction images; however, it helps to achieve faster phase recovery; (iii) the square root of the diffracted field intensity, calculated from upsampled lens-free images, is propagated back to the nanoaperture plane with an initial guess of zero phase. The object support (which is known a priori due to the precise fabrication of the aperture array) is then enforced at the nanoaperture plane by thresholding the intensity of the field, where the complex field outside the support is replaced with a uniform background intensity value. Next, the modified field at the nanoaperture plane is propagated back to the detector plane, creating a complex field with a non-zero twodimensional phase function. The amplitude of this complex field is replaced with the square root of the original recorded diffraction field intensity while leaving the new phase function untouched. After repeating this iterative process with approximately $N$ of $\sim 15$ cycles, the two-dimensional phase of the diffracted field incident on the detector array can be iteratively recovered from a single 

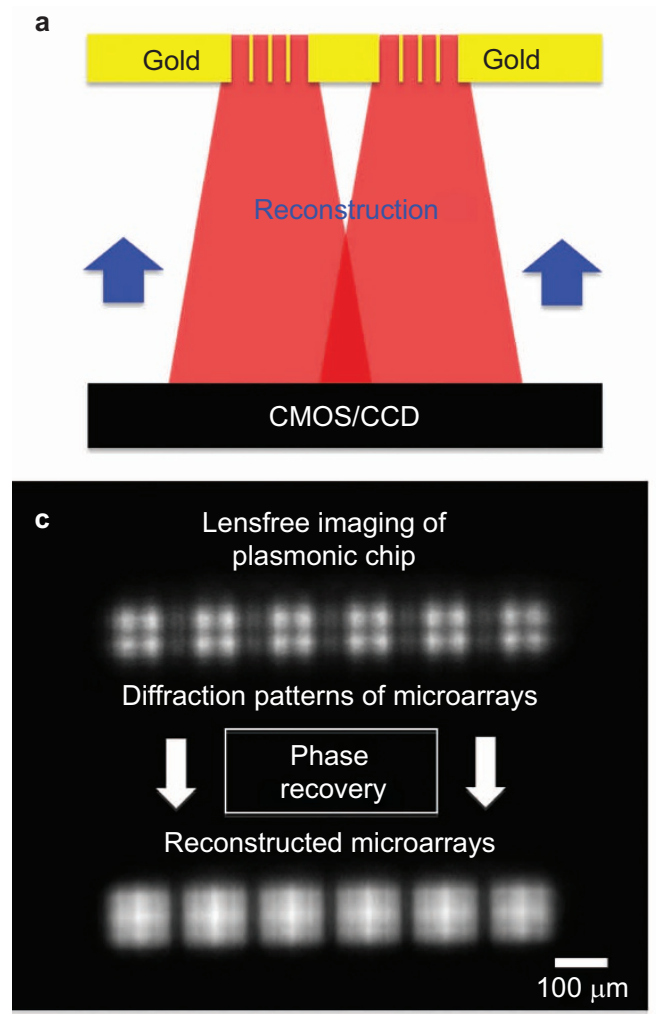

b

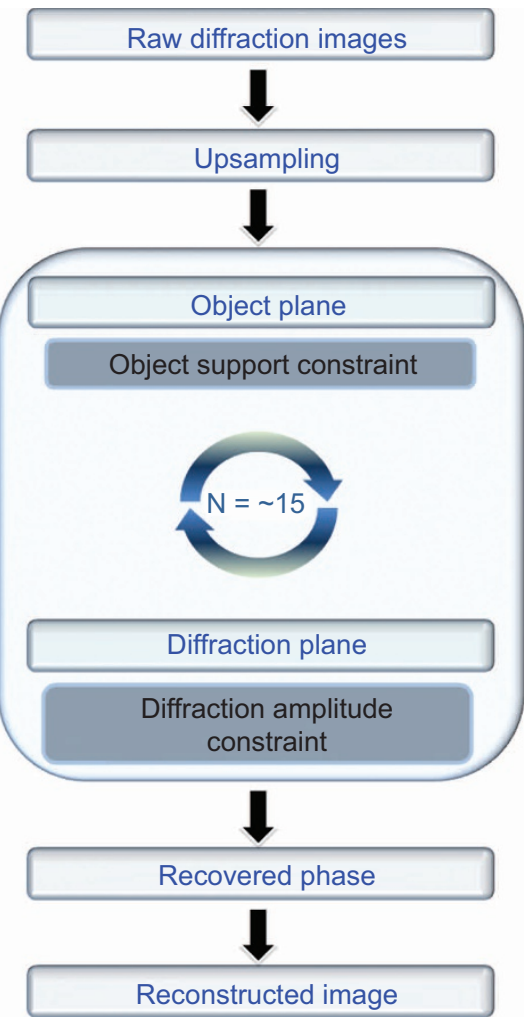

Figure 3 Numerical technique for the reconstruction of the diffraction images through a phase retrieval method. (a) Illustration of the partially overlapping diffraction patterns of the dense plasmonic microarrays at the detector/sampling plane due to our lens-free operation. (b) Workflow of the reconstruction procedure based on an iterative phase recovery algorithm. (c) Partially overlapping diffraction patterns of six different neighboring plasmonic microarray pixels, separated by $25 \mu \mathrm{m}$ edge-toedge distance, acquired by the lens-free imager at a propagation distance of $2 \mathrm{~mm}$ from the nanostructures to the sensor plane. The reconstructed images of the microarray pixels using the phase retrieval approach are also presented.

intensity image; ${ }^{65}$ and (iv) this complex field can then be backpropagated to the object/aperture plane, providing the reconstructed images of the plasmonic microarrays at the nanoaperture plane. To demonstrate the success of the above outlined phase recovery-based image reconstruction process, Figure $3 \mathrm{c}$ illustrates the diffraction patterns of six different neighboring plasmonic sensor pixels (separated by $25 \mu \mathrm{m}$ edge-to-edge distance) that are detected by the CMOS imager chip at a propagation distance of $2 \mathrm{~mm}$ from the nanostructures to the sensor plane, exhibiting partial spatial overlaps due to our lens-free operation. Employing the iterative reconstruction procedure discussed above, the diffracted lens-free images of the plasmonic sensor pixels can now be digitally focused onto the nanoaperture plane, removing the spatially overlapping parts of these images. This lens-free image reconstruction process is quite valuable especially for highly dense arrays of nanoapertures for increased multiplexing. However, we should also note that quantitative sensing information of the binding events could also be extracted from only the diffraction patterns of the same nanoapertures in case the physical separation between neighboring aperture regions is large, minimizing the intensity overlap at the detector/sampling plane. ${ }^{66}$ Using this phase recovery approach procedure, we can reduce the distance between individual sensory pixels even down to $2 \mu \mathrm{m} .{ }^{66} \mathrm{~A}$ single sensor with a size of $10 \mu \mathrm{m} \times 10 \mu \mathrm{m}$, consisting of more than 200 nanoholes (hole diameter $=200 \mathrm{~nm}$ and array period $600 \mathrm{~nm}$ ) should result in sufficient transmitted signal to be captured by the CMOS imager. ${ }^{84}$ Based on these numbers, employing a CMOS imager with an active area of $5.7 \mathrm{~mm} \times 4.3 \mathrm{~mm}$, we could image 170000 sensor pixels all in parallel, which is highly promising for high-throughput applications.

\section{RESULTS AND DISCUSSION}

Multiplexing performance of the lens-free detection platform

To evaluate the performance of our computational biosensing platform, we demonstrate multiplexed detection of protein mono- and bilayers. Figure $4 \mathrm{a}$ and $4 \mathrm{~b}$ show the schematic view and the diffraction patterns of the analyzed microarray pixels, respectively. Here, the microarray pixels enumerated from ' 1 ' to ' 6 ' correspond to the bare sensors, and ' $\mathrm{M}$ ' and 'B' correspond to the same sensors after introducing protein monolayer $(\mathrm{M})$ containing bovine serum albumin $(\mathrm{BSA}, 0.5 \% \mathrm{v} / \mathrm{v})$, and protein bilayer (B) containing $0.5 \mathrm{mg} \mathrm{mL}$ protein $\mathrm{A} / \mathrm{G}$ and $0.5 \mathrm{mg} \mathrm{mL} \mathrm{mL}^{-1}$ protein $\mathrm{IgG}$, respectively. ${ }^{91-94,115}$ Here, approximately $150 \mathrm{pL}$ protein solution is precisely introduced on the individual sensor pixel using a protein nanospotter. ${ }^{115}$ BSA has a molecular weight of $66 \mathrm{kDa}$ and forms a very thin layer on our plasmonic substrate, approximately $3 \mathrm{~nm} .{ }^{116,117}$ Figure $4 \mathrm{c}$ shows the transmission spectrum of our nanohole array before and after the presence of the protein layers. Initially, for the 6 bare pixels, the plasmonic mode has an average resonance wavelength of $\sim 690.3 \mathrm{~nm}$, with a standard deviation (s.d.) of $0.74 \mathrm{~nm}$. For the 3 pixels that are covered with protein monolayer, the plasmonic mode shifts to $696.5 \mathrm{~nm}$ (s.d. $=0.21 \mathrm{~nm}$ ) and for the remaining 3 pixels with protein bilayer, it shifts to $707.4 \mathrm{~nm}$ (s.d. $=0.77 \mathrm{~nm})$. As clearly seen by the lens-free diffraction pattern images in Figure $4 \mathrm{~b}$, monolayer spotted sensors look brighter than the bilayer spotted ones due to the additional $11 \mathrm{~nm}$ shift from the LED peak wavelength. Figure $4 \mathrm{~d}$ shows the statistical analysis of our plasmonic pixels, demonstrating a good correlation between the resonance wavelength shift and the relative intensity difference corresponding to mono- and bilayers. 

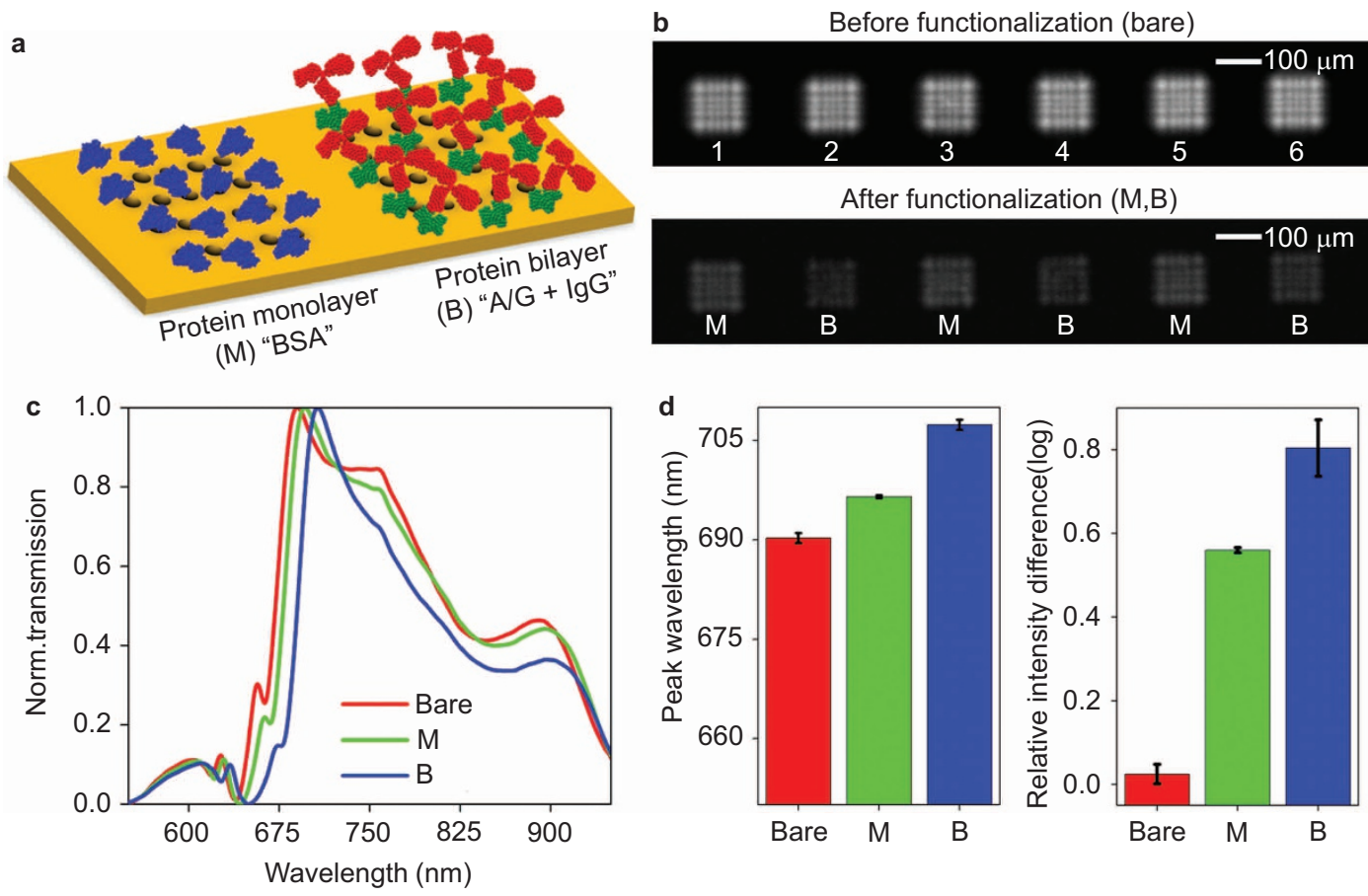

Figure 4 Multiplexing capability of the on-chip computational biosensing platform. (a) Schematic view and (b) the lens-free diffraction patterns of six different plasmonic sensors enumerated from ' 1 ' to ' 6 ' corresponding to bare sensors; and ' $\mathrm{M}$ ' and 'B' corresponding to the same sensors after introducing protein monolayer (M) containing BSA $(0.5 \% \mathrm{v} / \mathrm{v})$ and protein bilayer (B) containing $0.5 \mathrm{mg} \mathrm{mL}^{-1}$ protein $\mathrm{A} / \mathrm{G}$ and $0.5 \mathrm{mg} \mathrm{mL}^{-1}$ protein IgG, respectively. (c) Spectral transmission response of the plasmonic microarray pixels containing nanohole arrays before and after introducing the protein layers. (d) Statistical analysis of the plasmonic pixels for the peak wavelength shift calculated from optical spectrum analyzer measurements (left), and the relative intensity difference computed from the lens-free images (right), belonging to mono- and bilayers. BSA, bovine serum albumin.

\section{Quantitative analysis of binding events using lens-free on-chip detection}

Our on-chip plasmonic biosensing platform can also be utilized for quantitative analysis of binding events, providing precise concentration of target biomolecules bound to the plasmonic substrates. Towards this end, we demonstrate the quantification of a single-type of protein in a large variety of concentrations, spanning from microgram per milliliter to milligram per milliliter range. Furthermore, we image reference and target plasmonic microarray pixels simultaneously on the same chip to quantify the binding events on the target sensors, engineering a robust self-calibrated biodetection system that operates independent of the LED intensity variations from measurement to measurement. To illustrate this, Figure 5a shows lens-free diffraction patterns of six different sensors where (i) the pixels denoted by ' $M$ ' are the reference sensors and their spectral responses stay constant through the whole concentration experiment; and (ii) the pixels denoted by 'B' are the target sensors used for our concentration analysis. In these measurements, reference sensors are initially covered with BSA to block nonspecific binding events on these pixels, and the target sensors are covered with protein $\mathrm{A} / \mathrm{G}\left(\sim 4 \mathrm{mg} \mathrm{mL}^{-1}\right)$ for capturing protein IgG on the sensor surface. In more complex sample matrices, custom designed blocking agents can also be used, in a similar fashion, to reduce nonspecific molecular bindings. Note that the BSA and A/G pixels provided comparable level of spectral shift and transmitted signal intensity, indicating that similar amount of biomass bound to the reference and target pixels. Target pixels have then been processed with 8 different IgG concentrations, ranging from $3.9 \mu \mathrm{g} \mathrm{mL}^{-1}$ to $1000 \mu \mathrm{g} \mathrm{mL}^{-1}$. Figure $5 \mathrm{~b}$ shows the average spectral response, measured by an optical spectrum analyzer, of the reference and the target sensors for different protein concentrations demonstrating that the plasmonic mode consistently shifts to longer wavelengths as the concentration increases. The statistical analysis calculated from the optical spectrum analyzer measurements in Figure $5 \mathrm{c}$ shows that the difference in the average resonance shift value for the target pixels spanning a range from $2.95 \mathrm{~nm}$ to $27.1 \mathrm{~nm}$. In the figure inset, we show the relationship between the spectral shift in the plasmonic mode and the IgG concentration. In the low concentration range (e.g., 3.9-100 $\mu \mathrm{g} \mathrm{mL}^{-1}$ ), the plasmonic mode red-shifts linearly with increasing IgG concentration, whereas it shows minor variations at high concentrations $\left(250-1000 \mu \mathrm{g} \mathrm{mL}^{-1}\right)$ due to saturation of binding sites. Calculated from the lens-free images, the corresponding intensity difference analysis in Figure $5 \mathrm{~d}$ reveals a minimum detectable intensity difference of 0.024 (a.u.) as denoted by the red curve which corresponds to a minimum detectable wavelength shift of $3 \mathrm{~nm}$ for the transmission resonance from its initial position. Note that this $3 \mathrm{~nm}$ limit of detection can also be converted into a refractive index sensitivity of $621 \mathrm{~nm} \mathrm{RIU}{ }^{-1}$, corresponding to a minimum detectable refractive index change of $\sim 4 \times 10^{-3}$ RIU (see Supplementary Figs. S4 and S5 for the same analysis conducted using an optical spectrum analyzer). These results summarized in Figures 4 and 5 confirm that our plasmonic biosensing approach is highly promising for simultaneous detection of different biomolecules over a wide range of concentrations using the same field-portable and cost-effective handheld platform.

The presented computational high-throughput biosensing platform lends itself to a light-weight and compact point-of-care diagnostic tool that can potentially provide real-time results without the need for any trained professionals. Although such on-chip sensing 
a

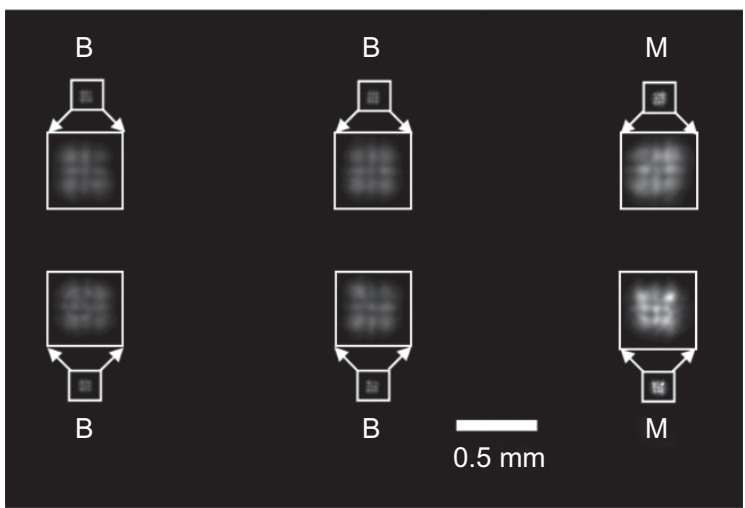

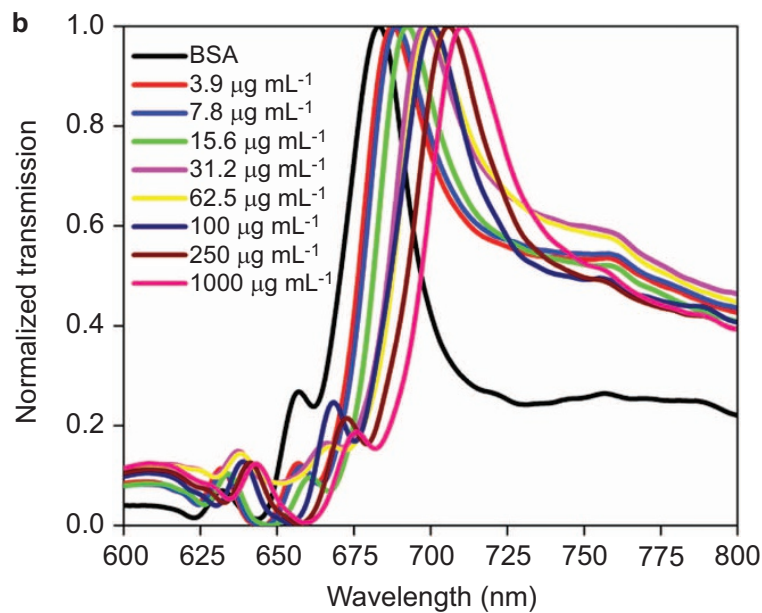

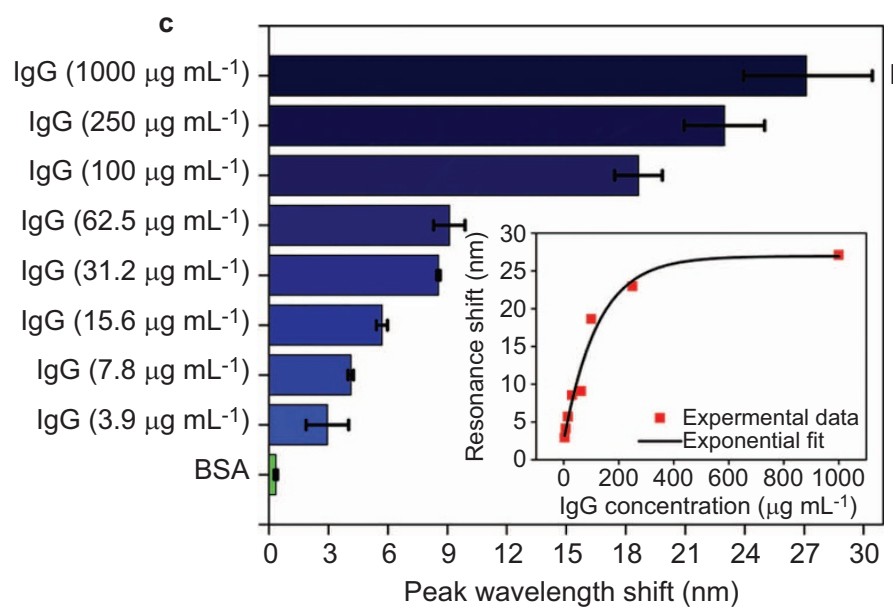

d

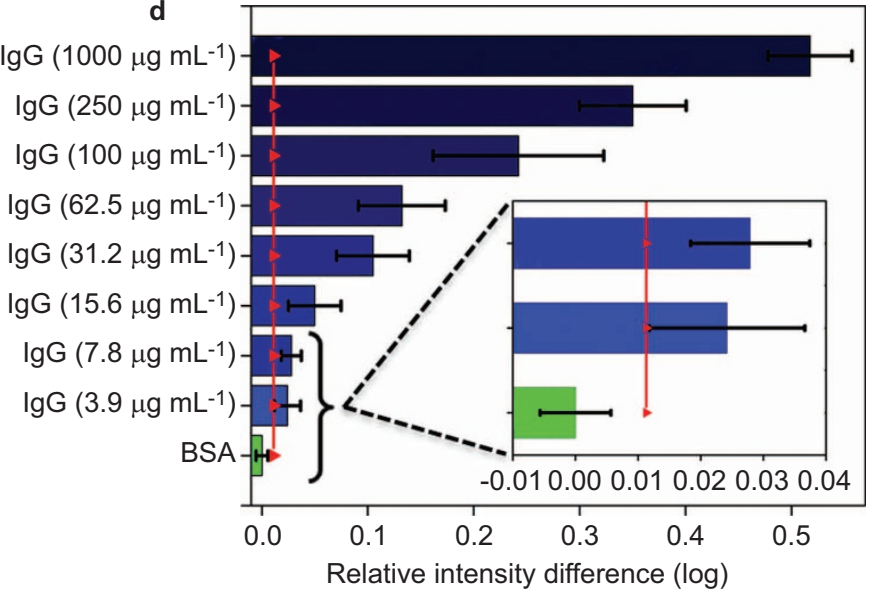

Figure 5 Quantification of a single-type protein concentration. (a) Lens-free diffraction patterns of the 2 reference and 4 target plasmonic microarray pixels are analyzed on the same chip to quantify the binding events on the target sensors, where reference and target sensors are covered with protein monolayers ( $\mathrm{M}$ ) containing BSA and protein bilayers (B) containing A/G and IgG, respectively. (b) Spectral response of the plasmonic sensors functionalized with different protein IgG concentrations, ranging from $3.9 \mu \mathrm{g} \mathrm{mL}^{-1}$ to $1000 \mu \mathrm{g} \mathrm{mL}{ }^{-1}$. Statistical analysis of (c) the peak wavelength shift in the plasmonic mode calculated from the optical spectrum analyzer measurements (figure inset: resonance shift in the plasmonic mode vs. IgG concentration) and (d) the corresponding intensity difference analysis determined from our lens-free images (figure inset: zoomed figure showing the detection limit). A minimum detectable intensity difference of 0.024 (a.u.), indicated by a red line, can be calculated by adding twice the standard deviation to the mean intensity difference value of the reference sensor containing BSA, which corresponds to a minimum detectable wavelength shift of $3 \mathrm{~nm}$. BSA, bovine serum albumin.

platform holds promise as a field-deployable and cost-effective handheld diagnostics device, especially for developing countries, it is important to note that our lens-free imaging platform has relatively higher detection limits (in the order of $\sim \mu \mathrm{g} \mathrm{mL}{ }^{-1}$ ) compared to other plasmonic sensing approaches such as conventional SPR-based platforms. In order to systematically improve our detection limits (down to $\mathrm{ng} \mathrm{mL}^{-1}$ ) we can explore several avenues: (i) spectrally narrow chip-based optoelectronic excitation sources, e.g., laser diodes or resonant-cavity enhanced LEDs can be employed to determine the minute spectral variations in the plasmonic modes; (ii) superior plasmonic designs achieving much sharper plasmonic resonances with stronger near-field enhancements, such as Fano resonant structures, ${ }^{94,118,119}$ can be implemented; (iii) the spectral shifts in the transmission resonance of the nanoapertures can be more sensitively tracked by acquiring multiple lens-free images (i.e., each with a different color LED). These lens-free frames can then be digitally merged, producing higher contrast differences between the reference and target images; ${ }^{84}$ (iv) better CMOS/CCD imagers, equipped with cooling circuits, can be employed in this plasmonic sensing platform achieving higher sensitivities; (v) advanced computational reconstruction approaches, e.g., based on convex optimization, ${ }^{120-123}$ can be applied to the diffraction images of the plasmonic chips; and (vi) nanoparticle based assays can be also functionalized on the same plasmonic substrates improving the binding sites of biomolecules, and further enhancing the contrast of the low-density biomolecules detected through improved lens-free diffraction patterns. A major advantage of using nanoparticles in this on-chip biosensing platform would be to increase the near-field interactions between the biomolecules and the surface plasmon waves up to the penetration depth of evanescent waves $(<$ $200 \mathrm{~nm}$ ). In our current plasmonic biosensing work, the binding events occur on a planar plasmonic substrate, limiting the near-field interactions only to the surface of the gold layer. Therefore, nanoparticles with diameters of $200-300 \mathrm{~nm}$ could provide additional three-dimensional binding sites, increasing the interactions of the biomolecules and surface plasmons. The proof of concept of such an approach is already illustrated in Supplementary Figs. S6 and S7, where streptavidin-coated nanoparticles bound to the plasmonic substrates can be detected using our on-chip biosensing platform, creating an enhanced contrast of the lens-free diffraction images after the binding events. Using a systematic investigation of the avenues 
discussed above, the detection limits of our computational plasmonic sensing platform can be improved down to $\mathrm{ng} \mathrm{mL}^{-1}$ range while still keeping the entire platform compact, light-weight, cost-effective and high-throughput for field medicine and point-of-care diagnostics.

\section{CONCLUSIONS}

In conclusion, we have demonstrated an on-chip computational biosensing approach that uniquely integrates plasmonic microarrays and a lens-free computational microscopy platform towards label-free, high-throughput and multiplexed detection of ultrathin layers of biomolecular binding events. This cost-effective and portable biosensing device weights $60 \mathrm{~g}$ and employs an off-the-shelf CMOS imager to record the lens-free diffraction patterns of plasmonic nanoaperture arrays that are illuminated by a quasimonochromatic plane wave from an LED which is tuned to the plasmonic resonances. Combining a sensitive plasmonic microarray substrate and lens-free computational imaging, our on-chip biosensing device can detect biomolecules with protein layer thicknesses down to $\sim 3 \mathrm{~nm}$. The presented computational biosensing approach, integrating large-scale plasmonic microarrays, could be especially useful for simultaneous detection of a large number of biomolecular interactions in field settings without the use of any bulky and costly instruments, creating a compact and highthroughput handheld technology for point-of-care and telemedicine applications.

\section{ACKNOWLEDGMENTS}

Altug Research Group acknowledges National Science Foundation (NSF) CAREER Award, Presidential Early Career Award for Scientist and Engineers (PECASE) ECCS-0954790, Office of Naval Research Young Investigator Award 11PR00755-00-P00001, NSF Engineering Research Center on Smart Lighting EEC-0812056, Massachusetts Life Sciences Center Young Investigator award and Ecole Polytechnique Federale de Lausanne. Ozcan Research Group acknowledges the support of PECASE, Army Research Office (ARO) Life Sciences Division, ARO Young Investigator Award, NSF CAREER Award, ONR Young Investigator Award and the National Institute of Health (NIH) Director's New Innovator Award DP2OD006427 from the Office of The Director, NIH and the NSF EFRI Award.

1 Wei F, Patel P, Liao W, Chaudhry K, Zhang L et al. Electrochemical sensor for multiplex biomarkers detection. Clin Cancer Res 2009; 15: 4446-4452.

2 Oikonomopoulou K, Li L, Zheng Y, Simon I, Wolfert RL et al. Prediction of ovarian cancer prognosis and response to chemotherapy by a serum-based multiparametric biomarker panel. Br J Cancer 2008; 99: 1103-1113.

3 Kozak KR, Su F, Whitelegge JP, Faull K, Reddy S et al. Characterization of serum biomarkers for detection of early stage ovarian cancer. Proteomics 2005; 5: 45894596.

4 Myers FB, Lee LP. Innovations in optical microfluidic technologies for point-of-care diagnostics. Lab Chip 2008; 8: 2015-2031.

5 Chin CD, Linder V, Sia SK. Lab-on-a-chip devices for global health: past studies and future opportunities. Lab Chip 2007; 7: 41-57.

6 Linder V. Microfluidics at the crossroad with point-of-care diagnostics. Analyst 2007; 132: 1186-1192.

7 Warsinke A. Point-of-care testing of proteins. Anal Bioanal Chem 2009; 393: 1393 1405.

8 Dungchai W, Chailapakul O, Henry CS. Electrochemical detection for paper-based microfluidics. Anal Chem 2009; 81: 5821-5826.

9 Jokerst JV, McDevitt JT. Programmable nano-bio-chips: multifunctional clinical tools for use at the point-of-care. Nanomedicine 2010; 5: 143-155.

10 Chin CD, Linder V, Sia SK. Commercialization of microfluidic point-of-care diagnostic devices. Lab Chip 2012; 12: 2118-2134.

11 Sia SK, Kricka LJ. Microfluidics and point-of-care testing. Lab Chip 2008; 8: 1982 1983.

12 Chin CD, Laksanasopin T, Cheung YK, Steinmiller D, Linder V et al. Microfluidicsbased diagnostics of infectious diseases in the developing world. Nat Med 2011; 17: 1015-1019.

13 Peeling RW, Mabey D. Point-of-care tests for diagnosing infections in the developing world. Clin Microbiol Infect 2010; 16: 1062-1069.
14 Goldberg BD, Nezam SM, Jillella P, Bouma BE, Tearney GJ. Miniature swept source for point of care optical frequency domain imaging. Opt Exp 2009; 17: 3619-3629.

15 Whitesides GM. The origins and future of microfluidics. Nature $2006 ; 442$ : 368-373

16 Miller AR, Davis GL, Oden ZM, Razavi MR, Fateh A et al. Portable, battery-operated, low-cost, bright field and fluorescence microscope. PLOS ONE 2010; 5: e11890 doi:10.1371/journal.pone.0011890.

17 Flusberg BA, Nimmerjahn A, Cocker ED, Mukamel EA, Barretto RP et al. High-speed, miniaturized fluorescence microscopy in freely moving mice. Nat Methods 2008; 5 : 935-938.

18 McCall B, Pierce M, Graviss EA, Richards-KortumR, Tkaczyk T. Toward a low-cost compact array microscopy platform for detection of tuberculosis. Tuberculosis 2011; 91: S54-S60.

19 Breslauer DN, Maamari RN, Switz NA, Lam WA, Fletcher DA. Mobile phone based clinical microscopy for global health applications. PLOS ONE 2009; 4: e6320. doi:10.1371/journal.pone.0006320.

20 Kim JS, Anderson GP, Erickson JS, Golden JP, Nasir M et al. Multiplexed detection of bacteria and toxins using a microflow cytometer. Anal Chem 2009; 81: 5426-5432.

21 Kiesel P, Bassler M, Beck M, Johnson N. Spatially modulated fluorescence emission from moving particles. App/ Phys Lett 2009; 94: 041107.

22 Daaboul GG, Yurt A, Zhang X, Hwang GM, Goldberg BB et al. High-throughput detection and sizing of individual low-index nanoparticles and viruses for pathogen identification. Nano Lett 2010; 10: 4727-4731.

23 Lu Y, Shi W, Qin J, Lin B. Low cost, portable detection of gold nanoparticle-labeled microfluidic immunoassay with camera cell phone. Electrophoresis 2009; 30: 579582.

24 Martinez AW, Phillips ST, Carrilho E, Thomas SW, Sindi H et al. Simple telemedicine for developing regions: camera phones and paper-based microfluidic devices for realtime, off-site diagnosis. Anal Chem 2008; 80: 3699-3707.

25 Pamplona V, Passos E, Zizka J, Oliveira M, Lawson E et al. CATRA: cataract probe with a lightfield display and a snap-on eyepiece for mobile phones. In: Proceedings of SIGGRAPH 2011; 12-15 December 2011; Hong Kong, China. ACM: New York, USA, $2011 ; 7: 1-47$.

26 Bourquin Y, Reboud J, Wilson R, Zhang Y, Cooper JM. Integrated immunoassay using tunable surface acoustic waves and lensfree detection. Lab Chip 2011; 11: 27252730.

27 Kostov Y, Sergeev N, Wilson S, Herold KE, Rasooly A. A simple portable electroluminescence illumination-based CCD detector. Methods Mol Biol 2009; 503: 259-272. doi:10.1007/978-1-60327-567-5_14.

28 Yang M, Sun S, Kostov Y, Rasooly A. An automated point-of-care system for immunodetection of staphylococcal enterotoxin B. Anal Biochem 2011; 416: 74-81.

29 Shen L, Hagen JA, Papautsky I. Point-of-care colorimetric detection with a smartphone. Lab Chip 2012; 12: 4240-4243.

30 Boyle DS, Hawkins KR, Steele MS, Singhal M, Cheng X. Emerging technologies for point-of-care CD4 T-lymphocyte counting. Trends Biotechnol 2012; 30: 45-54.

31 Weigl B, Domingo G, LaBarre P, Gerlach J. Towards non- and minimally instrumented, microfluidics-based diagnostic devices. Lab Chip 2008; 8: 1999-2014.

32 Soper SA, Brown K, Ellington A, Frazier B, Garcia-Manero G et al. Point-of-care biosensor systems for cancer diagnostics/prognostics. Biosens Bioelectron 2006; 21: 1932-1942.

33 Ellerbee A, Philips S, Siegel AC, Mirica KA, Martinez AW et al. Quantifying colorimetric assays in paper-based microfluidic devices by measuring the transmission of light through paper. Anal Chem 2009; 81: 8447-8452.

34 Sun S, Yang M, Kostov Y, Rasooly A. ELISA-LOC: lab-on-a-chip for enzyme-linked immunodetection. Lab Chip 2010; 10: 2093-2100.

35 Luppa PB, Muller C, Schlichtiger A, Schlebusch H. TrAC, point-of-care testing (POCT): current techniques and future perspectives. Trends Anal Chem 2011; 30: 887-898.

36 Zhu H, Isikman SO, Mudanyali O, Greenbaum A, Ozcan A. Optical imaging techniques for point-of-care diagnostics. Lab Chip 2013; 13: 51-67.

37 Mudanyali O, Tseng D, Oh C, Isikman SO, Sencan I et al. Compact, light-weight and cost-effective microscope based on lensless incoherent holography for telemedicine applications. Lab Chip 2010; 10: 1417-1428.

38 Mudanyali O, Dimitrov S, Sikora U, Padmanabhan S, Navruz I et al. Integrated rapiddiagnostic-test reader platform on a cellphone. Lab Chip 2012; 12: 2678-2686.

39 Coskun AF, Wong J, Khodadadi D, Nagi R, Tey A et al. A personalized food allergen testing platform on a cellphone. Lab Chip 2013; 13: 636-640.

40 Zhu H, Sencan I, Wong J, Dimitrov S, Tseng D et al. Cost-effective and rapid blood analysis on a cell-phone. Lab Chip 2013; 13: 1282-1288.

41 Makler MT, Palmer CJ, Ager AL. A review of practical techniques for the diagnosis of malaria. Ann Trop Med Parasitol 1998; 92: 419-433.

42 Ghosh KK, Burns LD, Cocker ED, Nimmerjahn A, Ziv Y et al. Miniaturized integration of a fluorescence microscope. Nat Methods 2011; 8: 871-878.

43 Smith ZJ, Chu K, Espenson AR, Rahimzadeh M, Gryshuk A et al. Cell-phone-based platform for biomedical device development and education applications. PLOS ONE 2011; 6: e17150. doi:10.1371/journal. pone.0017150.

44 Park S, Park M, Kang M. Super-resolution image reconstruction: a technical overview. IEEE Signal Process Mag 2003; 20: 21-36.

45 Isikman SO, Bishara W, Sikora U, Yaglidere O, Yeah J et al. Field-portable lensfree tomographic microscope. Lab Chip 2011; 11: 2222-2230.

46 Cui X, Lee LM, Heng X, Zhong W, Sternberg PW et al. Lensless high-resolution on-chip optofluidic microscopes for Caenorhabditis elegans and cell imaging. Proc Natl Acad Sci USA 2008; 105: 10670-10675. 
47 Zhu H, Sencan I, Wong J, Dimitrov S, Tseng D et al. Cost-effective and rapid blood analysis on a cell-phone. Lab Chip 2013; 13: 1282-1288.

48 Zhu H, Sikora U, Ozcan A. Quantum dot enabled detection of Escherichia coli using a cell-phone. Analyst 2012; 137: 2541-2544.

49 Greenbaum A, Sikora U, Ozcan A. Field-portable wide-field microscopy of dense samples using multi-height pixel super-resolution based lensfree imaging. Lap Chip 2012; 12: 1242-1245.

50 Zhu H, Mavandadi S, Coskun AF, Yaglidere O, Ozcan A. Optofluidic fluorescent imaging cytometry on a cell phone. Anal Chem 2011; 83: 6641-6647.

51 Bishara W, Sikora U, Mudanyali O, Ting-Wei S, Yaglidere 0 et al. Holographic pixel super-resolution in portable lensless on-chip microscopy using a fiber-optic array. Lab Chip 2011; 11: 1276-1279.

52 Zhu H, Yaglidere O, Su TW, Tseng D, Ozcan A. Cost-effective and compact wide-field fluorescent imaging on a cell-phone. Lab Chip 2011; 11: 315-322.

53 Tseng D, Mudanyali O, Oztoprak C, Isikman SO, Sencan I et al. Lensfree microscopy on a cellphone. Lab Chip 2010; 10: 1787-1792.

54 Shin D, Pierce MC, Gillenwater AM, Williams MD, Richards-Kortum R. A fiber optic fluorescence microscope using a consumer-grade digital camera for in vivo cellular imaging. PLoS ONE 2010; 5: e11218. doi:10.1371/journal.pone.0011218.

55 Fercher AF, Hitzenberger C, Juchem M. Measurement of intraocular optical distances using partially coherent laser light. J Mod Opt 1991; 38: 1327-1333.

56 Fercher AF, Hitzenberger CK, Kamp G, El-Zaiat SY. Measurement of intraocular distances by backscattering spectral interferometry. Opt Commun 1995; 117: 4348.

57 Boppart SA, Bouma BE, Pitris C, Tearney GJ, Fujimoto JG et al. Forward-imaging instruments for optical coherence tomography. Opt Lett 1997; 22: 1618-1620.

58 Liu L, Gardecki JA, Nadkarni SK, Toussaint JD, Yagi Y et al. Imaging the subcellular structure of human coronary atherosclerosis using micro-optical coherence tomography. Nat Med 2011; 17: 1010-1014.

59 Zheng K, Liu B, Huang C, Brezinski ME. Experimental confirmation of potential swept source optical coherence tomography performance limitations. App/ Opt 2008; 47: 6151-6158.

60 Jung W, Kim J, Jeon M, Chaney EJ, Stewart CN et al. Handheld optical coherence tomography scanner for primary care diagnostics. IEEE Trans Biomed Eng 2011; 58: 741-744.

61 Rosen J, Brooker G. Non-scanning motionless fluorescence three dimensional holographic microscopy. Nat Photonics 2008; 2: 190-195.

62 Barbastathis G, Balberg M, Brady DJ. Confocal microscopy with a volume holographic filter. Opt Lett 1999; 24: 811-813.

63 Piyawattanametha W, Cocker ED, Burns LD, Barretto RP, Jung JC et al. In vivo brain imaging using a portable $2.9 \mathrm{~g}$ two-photon microscope based on a microelectromechanical systems scanning mirror. Opt Lett 2009; 34: 2309-2311.

64 Greenbaum A, Luo W, Su TW, Göröcs Z, Xue L et al. Imaging without lenses: achievements and remaining challenges of wide-field on-chip microscopy. Nat Methods 2012; 9: 889-895.

65 Fienup JR. Reconstruction of an object from the modulus of its Fourier transform. Opt Lett 1978; 3: 27-29.

66 Khademhosseinieh B, Biener G, Sencan I, Su TW, Coskun AF et al. Lensfree sensing on a microfluidic chip using plasmonic nanoapertures. Appl Phys Lett 2010; 97: 221107.

67 Mudanyali O, McLeod E, Luo W, Greenbaum A, Coskun AF et al. Wide-field optical detection of nanoparticles using on-chip microscopy and self-assembled nanolenses. Nat Photonics 2013; 7: 247-254. doi:10.1038/nphoton.2012.337.

68 McLeod E, Luo W, Mudanyali O, Greenbaum A, Ozcan A. Toward giga-pixel nanoscopy on a chip: a computational wide-field look at the nano-scale without the use of lenses. Lab Chip 2013; 13: 2028-2035.

69 Lim LP, Lau NC, Garrett-Engele P, Grimson A, Schelter JM et al. Microarray analysis shows that some microRNAs downregulate large numbers of target mRNAs. Nature 2005; 433: 769-773.

70 Sassolas A, Leca-Bouvier BD, Blum LJ. DNA biosensors and microarrays. Chem Rev 2008; 108: 109-139.

71 MacBeath G, Schreiber SL. Printing proteins as microarrays for high-throughput function determination. Science 2000; 289: 1760-1763.

72 Ziauddin J, Sabatini DM. Microarrays of cells expressing defined cDNAs. Nature 2001; 411: 107-110.

73 Frank T, Tay S. Flow-switching allows independently programmable, extremely stable, high-throughput diffusion-based gradients. Lab Chip 2013; 13: 1273-1281.

74 Resch-Genger U, Grabolle M, Cavaliere-Jaricot S, Nitschke R, Nann T. Quantum dots versus organic dyes as fluorescent labels. Nat Methods 2008; 5: 763-775.

75 Zhang L, Hurek T, Reinhold-Hurek B. Position of the fluorescent label is a crucial factor determining signal intensity in microarray hybridizations. Nucleic Acids Res 2005; 33: e166. doi: 10.1093/nar/gni156.

76 Gruber HJ, Hahn CD, Kada G, Riener CK, Harms GS et al. Anomalous fluorescence enhancement of $\mathrm{Cy} 3$ and Cy3.5 versus anomalous fluorescence loss of Cy 5 and Cy 7 upon covalently linking to IgC and noncovalent binding to avidin. Bioconjugate Chem 2000; 11: 696-704.

77 Bunimovich YL, Shin YS, Yeo WS, Amori M, Kwong G et al. Quantitative real-time measurements of DNA hybridization with alkylated nonoxidized silicon nanowires in electrolyte solution. J Am Chem Soc 2006; 128: 16323-16331.

78 Fritz J, Baller M, Lang HP, Rothuizen $\mathrm{H}$, Vettiger $\mathrm{P}$ et al. Translating biomolecular recognition into nanomechanics. Science 2000; 288: 316-318.
79 Savran CA, Knudsen SM, Ellington AD, Manalis SR. Micromechanical detection of proteins using aptamer-based receptor molecules. Anal Chem 2004; 76: 31943198.

80 Fan X, White IM, Shopova SI, Zhu H, Suter JD et al. Sensitive optical biosensors for unlabeled targets: a review. Anal Chim Acta 2008; 620: 8-26.

81 Cubukcu E, Degirmenci F, Kocabas C, Zimmler MA, Rogers JA et al. Aligned carbon nanotubes as polarization-sensitive molecular near-field detectors. Proc Natl Acad Sci USA 2009; 106: 2495-2499.

82 Rudenko MI, Kuhn S, Lunt EJ, Deamer DW, Hawkins AR et al. Ultrasensitive Q $\beta$ phage analysis using fluorescence correlation spectroscopy on an optofluidic chip. Biosens Bioelectron 2009; 24: 3258-3263.

83 Kinkhabwala A, Yu Z, Fan S, Avlasevich Y, Mullen K et al. Large single-molecule fluorescence enhancements produced by a bowtie nanoantenna. Nat Photonics 2009; 3: 654-657.

84 Chang TY, Huang M, Yanik AA, Tsai HY, Shi P et al. Large-scale plasmonic microarrays for label-free high-throughput screening. Lab Chip 2011; 11: 3596-3602.

85 Icoz K, Savran CA. Nanomechanical biosensing with immunomagnetic separation. Appl Phys Lett 2010; 97: 123701.

86 Cooper MA. Optical biosensor in drug discovery. Nat Rev Drug Discovery 2002; 1: 515-528.

87 Homola J, Vaisocherova H, Dostalek J, Piliarik M. Multi-analyte surface plasmon resonance biosensing. Methods 2005; 37: 26-36.

88 Pryce IM, Kelaita YA, Aydin K, Briggs RM, Atwater HA. Compliant metamaterials for resonantly enhanced infrared absorption spectroscopy and refractive index sensing. ACS Nano 2011; 5: 8167-8174.

89 Kabashin AV, Nikitin PI. Interferometer based on a surface-plasmon resonance for sensor applications. Quantum Electron 1997; 27: 653-654.

90 Grigorenko AN, Nikitin PI, Kabashin AV. Phase jumps and interferometric surface plasmon resonance imaging. Appl Phys Lett 1999; 75: 3917-3919.

91 Yanik AA, Kamohara O, Artar A, Geisbert TW, Connor JH et al. An optofluidic nanoplasmonic biosensor for direct detection of live viruses from biological media. Nano Lett 2010; 10: 4962-4969.

92 Yanik AA, Cetin AE, Huang M, Artar A, Mousavi SH et al. Seeing protein monolayers with naked eye through plasmonic Fano resonances. Proc Natl Acad Sci USA 2011; 108: 11784-11789.

93 Adato R, Yanik AA, Amsden JJ, Kaplan DL, Omenetto FG et al. Ultra-sensitive vibrational spectroscopy of protein monolayers with plasmonic nanoantenna arrays. Proc Natl Acad Sci USA 2009; 106: 19227-19232.

94 Cetin AE, Altug H. Fano resonant ring/disk plasmonic nanocavities on conducting substrates for advanced biosensing. ACS Nano 2012; 6: 9989-9995.

95 Salvador JP, Kreuzer MP, Quidant R, Badenes G, Marco MP. Nanobiosensors for in vitro and in vivo analysis of biomolecules. Methods Mol Biol 2012; 811: 207221.

96 Zhang W, Huang L, Santschi C, Martin OJ. Trapping and sensing $10 \mathrm{~nm}$ metal nanoparticles using plasmonic dipole antennas. Nano Lett 2010; 10: 1006-1011.

97 Shegai T, Johansson P, Langhammer C, Käll M. Directional scattering and hydrogen sensing by bimetallic Pd-Au nanoantennas. Nano Lett 2012; 12: 2464-2469.

98 Hao F, Sonnefraud Y, van Dorpe P, Maier SA, Halas NJ et al. Symmetry breaking in plasmonic nanocavities: subradiant LSPR sensing and a tunable Fano resonance. Nano Lett 2008; 8: 3983-3988.

99 Liu N, Weiss T, Mesch M, Langguth L, Eigenthaler U et al. Planar metamaterial analogue of electromagnetically induced transparency for plasmonic sensing. Nano Lett 2010; 10: 1103-1107.

100 Gao H, McMahon JM, Lee MH, Henzie J, Gray SK et al. Rayleigh anomaly-surface plasmon polariton resonances in palladium and gold subwavelength hole arrays. Opt Express 2009; 17: 2334-2340.

101 Otte MA, Sepúlveda B, Ni W, Juste JP, Liz-Marzán LM et al. Identification of the optimal spectral region for plasmonic and nanoplasmonic sensing. ACS Nano 2010; 4: 349-357.

102 Schmidt MA, Lei DY, Wondraczek L, Nazabal V, Maier SA. Hybrid nanoparticlemicrocavity-based plasmonic nanosensors with improved detection resolution and extended remote-sensing ability. Nat Commun 2012; 3: 1108.

103 Im H, Bantz KC, Lee SH, Johnson TW, Haynes CL et al. Self-assembled plasmonic nanoring cavity arrays for SERS and LSPR biosensing. Adv Mater 2013; 25: 26782685.

104 Ebbesen TW, Lezec HJ, Ghaemi HF, Thio T, Wolff PA. Extraordinary optical transmission through sub-wavelength hole arrays. Nature 1998; 391: 667-669.

105 Rindzevicius T, Alaverdyan Y, Dahlin A, Hook F, Sutherland DS et al. Plasmonic sensing characteristics of single nanometric holes. Nano Lett 2005; 5: 2335-2339.

106 Yanik AA, Huang M, Artar A, Chang TY, Altug H. Integrated nanoplasmonicnanofluidic biosensors with targeted delivery of analytes. Appl Phys Lett 2010; 96: 021101.

107 Tetz KA, Pang L, Fainman Y. High-resolution surface plasmon resonance sensor based on linewidth-optimized nanohole array transmittance. Opt Lett 2006; 31: 15281530.

108 Barnes WL, Dereux A, Ebbesen TW. Surface plasmon subwavelength optics. Nature 2003; 424: 824-830.

109 Khanikaev AB, Mousavi SH, Shvets G, Kivshar YS. One-way extraordinary optical transmission and nonreciprocal spoof plasmons. Phys Rev Lett 2010; 105: 126804.

110 Eftekhari F, Escobedo C, Ferreira J, Duan X, Girotto EM et al. Nanoholes as nanochannels: flow-through plasmonic sensing. Anal Chem 2009; 81: 4308-4311. 
111 Artar A, Yanik AA, Altug H. Fabry-Perot nanocavities in multilayered crystals for enhanced biosensing. Appl Phys Lett 2009; 95: 051105.

112 Lalanne P, Rodier JC, Hugonin JP. Surface plasmons of metallic surfaces perforated by nanohole arrays. J Opt A-Pure Appl Opt 2005; 7: 422-426.

113 Cetin AE, Yanik AA, Yilmaz C, Somu S, Busnaina AA et al. Monopole antenna arrays for optical trapping, spectroscopy and sensing. Appl Phys Lett 2011; 98: 111110.

114 Goodman JW. Introduction to Fourier Optics. New York: McGraw-Hill; 1968.

115 Huang M, Galarreta B, Artar A, Adato R, Aksu S et al. Reusable nanostencils for creating multiple biofunctional molecular nanopatterns on polymer substrate. Nano Lett 2012; 12: 4817-4822.

116 Wright AK, Thompson MR. Hydrodynamic structure of bovine serum albumin determined by transient electric birefringence. Biophys J 1975; 15: 137-141.

117 Figueira VB, Jones JP. Viscoelastic study of the adsorption of bovine serum albumin on gold and its dependence on $\mathrm{pH}$. J Colloid Interface Sci 2008; 325: 107-113.

118 Wu CW, Khanikaev AB, Adato R, Arju N, Yanik AA et al. Fano-resonant asymmetric metamaterials for ultrasensitive spectroscopy and identification of molecular monolayers. Nat Mater 2012; 11: 69-75.
119 Artar A, Yanik A, Altug H. Directional double Fano resonances in plasmonic heterooligomers. Nano Lett 2011; 11: 3694-3700.

120 Kim SJ, Koh K, Lustig M, Boyd S, Gorinevsky D. An interior-point method for largescale $I_{1}$-regularized least squares. IEEE J Sel Topics Signal Process 2007; 1: 606617.

121 Donoho DL. Compressed sensing. IEEE Trans Inf Theory 2006; 52: 1289-1306.

122 Candès EJ, Romberg JK, Tao T. Stable signal recovery from incomplete and inaccurate measurements. Comm Pure Appl Math 2006; 59: 1207-1223.

123 Conchello JA. Superresolution and convergence properties of the expectationmaximization algorithm for maximum-likelihood deconvolution of incoherent images. J Opt Soc Am A Opt Image Sci Vis 1998; 15: 2609-2619.

(c) (i) $\odot$ This work is licensed under a Creative Commons Attribution-

NonCommercial-NoDerivs Works 3.0 Unported license. To view a copy of this license, visit http://creativecommons.org/licenses/by-nc-nd/3.0

Supplementary Information for this article can be found on Light: Science \& Applications's website (http://www.nature.com/lsa/). 